\title{
Pituitary-adrenal responses following major abdominal
} surgery

\author{
Ioanna Dimopoulou ${ }^{1}$, Marinella Tzanela ${ }^{2}$, Dimitra Vassiliadi ${ }^{2}$, Irini Mavrou ${ }^{1}$, \\ Petros Kopterides ${ }^{1}$, Stylianos Orfanos ${ }^{1}$, Anastasia Kotanidou ${ }^{3}$, \\ Styliani Kontogiannopoulou ${ }^{4}$, Spyridon Vasdekis ${ }^{5}$, Iraklis Tsangaris ${ }^{1}$, \\ Apostolos Armaganidis ${ }^{1}$, Anastasios Macheras ${ }^{5}$, Ioannis Ilias ${ }^{6}$, \\ Georgia Kostopanagiotou ${ }^{4}$, Stylianos Tsagarakis ${ }^{7}$
}

${ }^{1} 2^{\text {nd }}$ Department of Critical Care Medicine, "Attikon" Hospital, Athens University School of Medicine, ${ }^{2}$ Department of Endocrinology, ${ }^{3} 1^{\text {st }}$ Department of Critical Care Medicine, "Evangelismos" General Hospital, ${ }^{4} 2^{\text {nd }}$ Department of Anesthesiology, ${ }^{5} 3^{\text {rd }}$ Department of Surgery, "Attikon" Hospital, Athens University School of Medicine, ${ }^{6}$ Department of Endocrinology, "Elena Venizelou" Hospital, ${ }^{7}$ Department of Endocrinology, "Athens Polyclinic" Hospital, Athens, Greece

\begin{abstract}
OBJECTIVE: Perioperative pituitary-adrenal responses after major abdominal surgery have not been well characterized. The aim of the present study was to further clarify perioperative pituitary-adrenal responses. DESIGN: Thirty-six patients $(20$ men), aged $68 \pm 10$ years (mean \pm SD), undergoing major abdominal operations were studied. Total cortisol (TC), Adrenocorticotropic Hormone (ACTH), corticosteroid-binding globulin (CBG), dehydroepiandrosterone (DHEA) and its sulphate (DHEAS) were measured preoperatively (PreOp), on the day of surgery (DoS) and on the first and second postoperative days (PoD1 and PoD2). The free cortisol index (FCI) was also calculated. In addition, patients underwent a low-dose ( $1 \mu \mathrm{g})$ ACTH stimulation test, preoperatively and on PoD1, to assess adrenal responsiveness to ACTH. RESULTS: TC, FCI, ACTH and DHEA were increased, while DHEAS and CBG declined on DoS compared to PreOp levels. On PoD1, plasma ACTH was lower compared to the preoperative levels, FCI remained elevated, DHEA, DHEAS and TC returned to their baseline values, while CBG was still low. On PoD2, ACTH and CBG were low and FCI returned to its PreOp levels. Postoperatively, stimulated FCI was higher compared to the PreOp value. CONCLUSION: At an early phase following major surgery, elevated cortisol is associated with high ACTH. Despite HPA activation and a concomitant rise in DHEA levels, DHEAS declines. Later on, a remarkable dissociation between ACTH (low) and cortisol (high) is observed, which is attributed, at least in part, to increased adrenal responsiveness to ACTH.
\end{abstract}

Key words: Adrenal androgens, Corticotropin, DHEAS, Free cortisol index, Major surgery, Pituitary adrenal responses, Surgical stress 


\section{INTRODUCTION}

Major surgery, like any critical situation, activates the hypothalamic-pituitary-adrenal (HPA) axis mainly through the secretion of the hypothalamic corticotropin-releasing hormone ( $\mathrm{CRH}) . \mathrm{CRH}$ stimulates pituitary Adrenocorticotropic Hormone (ACTH), which in turn stimulates cortisol production in the adrenal cortex. ${ }^{1}$ In general, the degree and duration of hormonal changes after surgery, including HPA axis alterations, depend on the severity of the surgical trauma. ${ }^{2}$ In the vast majority of studies investigating the stress response to surgery, only total cortisol (TC) level was measured; in contrast, little is known about free serum cortisol, ${ }^{3-5}$ which, in fact, is the biologically active hormone. ${ }^{6}$ Furthermore, the mechanisms responsible for postoperative hypercortisolemia remain incompletely understood; in this regard, ACTHdependent along with non-ACTH-driven pathways have been implicated. ${ }^{7}$

The adrenal cortex is also the primary source of circulating adrenal androgens, such as dehydroepiandrosterone (DHEA) and its sulphated ester DHEAS. Under normal circumstances, DHEA and DHEAS rise in synchrony with cortisol in response to ACTH. ${ }^{8}$ In critically ill patients, however, dissociation between DHEAS (low) and cortisol (high) has been described. ${ }^{9}, 10$ In fact, the finding of low DHEAS has been considered as indicating an exhausted adrenal reserve..$^{10}$ It remains to be elucidated whether DHEA administration may be beneficial to critically ill patients. ${ }^{11}$ Measurements of DHEA levels, the bioactive component of adrenal androgens, have been presented in only a limited number of reports in critically ill septic patients and the results are controversial. ${ }^{12,13}$ Furthermore, DHEA/DHEAS system alterations, following major surgery, are variable..$^{14-17}$

In the present study we aimed to gain a better understanding of the HPA axis alterations in patients undergoing elective, major surgery, with no evidence of preoperative HPA axis dysfunction. To this end, ACTH, TC, cortisol-binding globulin (CBG), DHEA and DHEAS were measured perioperatively. The free cortisol index (FCI), reflecting serum free cortisol levels, ${ }^{18}$ was calculated. To further elucidate the mechanisms controlling cortisol changes, the responsiveness of the adrenal to stimulation with exogenously administered synthetic ACTH was in- vestigated pre- and postoperatively.

\section{SUBJECTS AND METHODS}

\section{Patients}

This prospective study included all patients undergoing major, elective abdominal surgery over a five-month period in a tertiary care academic department of surgery. Exclusion criteria were: neutropenia $\left(\leq 500\right.$ neutrophils $\left./ \mathrm{mm}^{3}\right)$; HIV infection; perioperative administration of corticosteroids, etomidate and dopamine, since the former interfere with HPA axis function and the latter suppresses DHEAS levels; ${ }^{7}$ history of endocrine disorder; preoperative HPA axis dysfunction, defined as preoperative stimulated cortisol less than $496 \mathrm{nmol} / \mathrm{L}$ after the administration of 1 $\mu \mathrm{g}$ of synthetic ACTH (low-dose stimulation test) ${ }^{19-23}$ and preoperative liver or renal dysfunction, as judged by routine laboratory tests. The study was approved by the hospital's Ethics Committee and informed consent was obtained from each patient.

During the study period, 36 patients (20 males) with a mean \pm SD age of $68 \pm 10$ years fulfilled the inclusion criteria. All patients were operated on under general anesthesia with sevoflurane. The patients underwent surgery for abdominal aorta aneurysm repair $(n=7)$ or for malignant gastrointestinal tumors [operations included colectomy $(n=17)$, total gastrectomy $(n=7)$ and Whipple's pancreatectomy $(\mathrm{n}=5)]$. Median duration of surgery was $150 \mathrm{~min}$ (range: 60 to $440 \mathrm{~min}$ ). Overall, four patients died due to postoperative complications.

\section{Methods}

Baseline blood sampling was performed preoperatively (PreOp; $24 \mathrm{~h}$ before surgery, at 08:00), on the day of surgery (DoS) immediately after the termination of the operation in the recovery room and on the first and second postoperative days (PoD1 and PoD2, respectively) at 08:00, to measure TC, ACTH, CBG, DHEA and DHEAS. The free cortisol index (FCI) was calculated as previously described, i.e. FCI $=$ serum $\mathrm{TC} / \mathrm{CBG} .^{5}$

Immediately after baseline sampling, patients underwent a low-dose stimulation test on two occasions: first, $24 \mathrm{~h}$ before surgery; and second, on PoD1. Briefly, $1 \mu \mathrm{g}$ of freshly prepared tetracosactrin 1-24 
(Synacthene, Novartis, Basel, Switzerland) $)^{19-23}$ was injected intravenously and $30 \mathrm{~min}$ later a blood sample was obtained to measure stimulated cortisol.

\section{Assays}

Serum TC was determined by an immunofluorometric assay (Chiron Corporation, East Walpole, MA, USA). ACTH was measured by an immunoradiometric assay (Nichols Institute Diagnostics, San Juan Capistrano, CA, USA). DHEA was measured by radioimmunoassay (DSL Systems Laboratories, Webster Inc., TX, USA). DHEAS was measured also by radioimmunoassay (Coat-A-Count, Diagnostic Products Corporation, CA, USA). CBG was determined by a radioimmunometric assay (DRG International, Inc., USA).

\section{Statistical analysis}

Data are presented as mean \pm SD or 1 st quartile/median/3nd quartile. Serial data were analysed by repeated measures analysis of variance on ranks followed by Tukey's test for specific comparisons. The correlations of TC and CBG were assessed with Pearson's correlation coefficient. The comparison of stimulated FCI before and after the operation was performed with the signed rank test. A $p<0.050$ was considered statistically significant.

\section{RESULTS}

There were significant overall perioperative changes in total cortisol $(p=0.005)$, ACTH $(p<0.001)$, FCI $(\mathrm{p}=0.028)$ and DHEA (Table 1). Elevation in TC was observed only on DoS. CBG levels decreased on DoS and remained lower than preoperatively on PoD1 and PoD2. On DoS, FCI, ACTH and DHEA increased, while DHEAs declined compared to the preoperative values. On PoD1, both DHEA and DHEAS returned almost to their baseline levels, and FCI remained elevated. On PoD2, FCI had returned to its baseline values. CBG correlated with TC $(r=0.498, p=0.002)$ on DoS, but not on PreOp ( $\mathrm{r}=0.181, \mathrm{p}=0.306)$, PoD1 $(\mathrm{r}=0.286, \mathrm{p}=0.089)$ or PoD2 $(\mathrm{r}=0.079, \mathrm{p}=0.649)$. Stimulated FCI to synthetic ACTH on PoD1 was higher compared to its preoperative value $(\mathrm{p}=0.010)$ (Figure 1; Table 2).

In the 4 patients who died, ACTH, cortisol and FCI were higher compared to the other patients on
Table 1. Descriptive statistics for corticotropin (ACTH), total cortisol, cortisol-binding globulin, free cortisol index, dehydroepiandrosterone (DHEA) and dehydroepiandrosterone sulphate (DHEAS) preoperatively (PreOp), on the day of surgery (DoS) and on postoperative days 1 and 2 (PoD1, PoD2). Asterisks denote Tukey's test $\mathrm{p}<0.05$ compared to PreOp values - see text for details; P25/P50/P75: $1^{\text {st }}$ quartile/median $/ 3^{\text {rd }}$ quartile.

\begin{tabular}{|c|c|c|c|}
\hline & & Mean \pm SD & P25/P50/P75 \\
\hline \multirow{4}{*}{$\begin{array}{l}\mathrm{ACTH} \\
(\mathrm{pmol} / \mathrm{L})\end{array}$} & PreOp & $3.60 \pm 3.43$ & $1.42 / 2.40 / 4.31$ \\
\hline & DoS* & $23.16 \pm 40.21$ & $1.94 / 4.29 / 25.16$ \\
\hline & PoD1 & $2.63 \pm 2.59$ & $1.29 / 2.13 / 2.62$ \\
\hline & PoD2 & $1.93 \pm 1.76$ & $0.72 / 1.64 / 2.24$ \\
\hline \multirow{4}{*}{$\begin{array}{l}\text { Total } \\
\text { Cortisol } \\
(\mathrm{nmol} / \mathrm{L})\end{array}$} & PreOp & $410.94 \pm 132.08$ & $302.80 / 405.57 / 454.54$ \\
\hline & DoS* & $506.43 \pm 297.92$ & $315.90 / 496.62 / 645.61$ \\
\hline & PoD1 & $452.86 \pm 344.48$ & $264.17 / 339.36 / 515.24$ \\
\hline & PoD2 & $367.49 \pm 156.42$ & $289.69 / 353.15 / 402.81$ \\
\hline \multirow{4}{*}{$\begin{array}{l}\text { Cortisol } \\
\text { Binding } \\
\text { Globulin } \\
(\mathrm{nmol} / \mathrm{L})\end{array}$} & PreOp & $717.51 \pm 296.78$ & $536.87 / 730.15 / 896.58$ \\
\hline & DoS* & $474.24 \pm 181.69$ & $375.81 / 476.74 / 568.01$ \\
\hline & PoD1* & $511.65 \pm 183.98$ & $407.81 / 515.40 / 573.16$ \\
\hline & PoD2* & $1702.91 \pm 6806.14$ & $450.97 / 569.09 / 631.36$ \\
\hline \multirow{4}{*}{$\begin{array}{l}\text { Free } \\
\text { Cortisol } \\
\text { Index } \\
\text { (unit-free) }\end{array}$} & PreOp & $0.57 \pm 0.45$ & $0.56 / 0.55 / 0.51$ \\
\hline & DoS* & $1.07 \pm 1.64$ & $0.84 / 1.04 / 1.13$ \\
\hline & PoD1* & $0.88 \pm 1.87$ & $0.65 / 0.66 / 0.91$ \\
\hline & PoD2 & $0.73 \pm 0.42$ & $0.64 / 0.62 / 0.64$ \\
\hline \multirow{4}{*}{$\begin{array}{l}\text { DHEA } \\
(\mathrm{nmol} / \mathrm{L})\end{array}$} & PreOp & $190.04 \pm 54.62$ & $88.45 / 150.12 / 263.99$ \\
\hline & DoS* & $340.88 \pm 163.82$ & $139.31 / 305.13 / 552.51$ \\
\hline & PoD1 & $160.93 \pm 56.26$ & $95.43 / 139.73 / 199.42$ \\
\hline & PoD2 & $171.24 \pm 52.39$ & $101.57 / 155.13 / 210.66$ \\
\hline \multirow{4}{*}{$\begin{array}{l}\text { DHEAS } \\
(\mu \mathrm{mol} / \mathrm{L})\end{array}$} & PreOp & $2.54 \pm 1.91$ & $1.18 / 2.00 / 3.53$ \\
\hline & DoS* & $1.77 \pm 1.20$ & $0.72 / 1.59 / 2.88$ \\
\hline & PoD1 & $2.33 \pm 1.58$ & $1.39 / 2.03 / 2.90$ \\
\hline & PoD2 & $2.20 \pm 1.34$ & $1.31 / 2.01 / 2.72$ \\
\hline
\end{tabular}

PoD1 (data not shown).

\section{DISCUSSION}

The present study investigated the perioperative HPA axis changes in patients undergoing major surgery. Two distinct patterns were noted: during the immediate postoperative phase, FCI, ACTH and DHEA rose, while DHEAS declined; on PoD1, FCI declined but remained higher than preoperative levels, whereas ACTH returned to preoperative levels. On PoD2, FCI also returned to its preoperative values.

The HPA axis is activated following major operations; however, the duration of this activation remains 


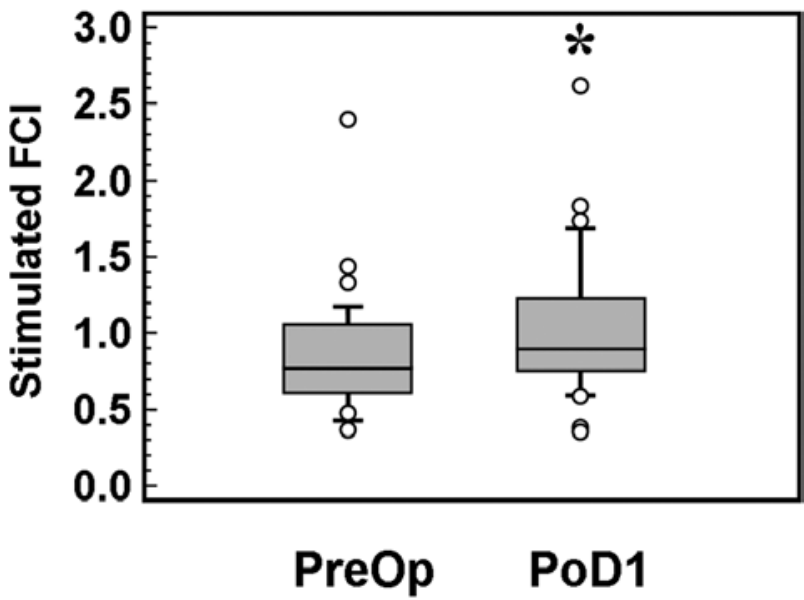

Figure 1. Stimulated free cortisol index to $1 \mu \mathrm{g}$ of corticotropin preoperatively (PreOp) and on postoperative day 1 (PoD1). Asterisks denote Tukey's test $p<0.05$ compared to PreOp values.

Table 2. Descriptive statistics for stimulated free cortisol index to $1 \mu \mathrm{g}$ of corticotropin preoperatively (PreOp) and on postoperative day 1 (PoD1). Asterisks denote Tukey's test $\mathrm{p}<0.05$ compared to PreOp values - see text for details; P25/P50/P75: $1^{\text {st }}$ quartile/ median $/ 3^{\text {rd }}$ quartile.

\begin{tabular}{lccc}
\hline & & Mean+SD & P25/P50/P75 \\
\hline $\begin{array}{l}\text { Free Cortisol Index } \\
\text { (unit-free) }\end{array}$ & PreOp & $0.93+0.62$ & $0.81 / 0.86 / 1.00$ \\
\cline { 2 - 4 } & PoD1 $^{*}$ & $1.23+1.77$ & $1.11 / 1.12 / 1.21$ \\
\hline
\end{tabular}

controversial. In this regard, some studies suggest that hormone levels return to normal within 24 hours post-surgery, ${ }^{24}$ while most indicate that cortisol may remain high for up to three days., ${ }^{3,25-27}$ In humans, the liver-derived CBG constitutes the major carrier protein for serum cortisol. ${ }^{6,7}$ Acute exposure to a stressor, such as septic shock or trauma, decreases serum CBG levels. ${ }^{28}$ In the face of changing CBG levels, it is unlikely that TC accurately reflects HPA axis activity. The FCI takes into account CBG alterations and has been found to correlate with serum free cortisol. ${ }^{18}$ By using this index, we found that in surgical patients with intact preoperative adrenal function, the HPA axis recovers within 24 hours after surgery. It remains uncertain whether glucocorticoidtreated patients need cortisol supplementation or their current maintenance dose to tolerate a surgical procedure..$^{29}$ Nevertheless, our results suggest that if in such patients perioperative glucocorticoid coverage is decided, this should be of a shorter duration than previously recommended. ${ }^{30}$

In most studies on stress response to surgery, only cortisol has been measured, despite the fact that $\mathrm{ACTH}$, the main regulator of glucocorticoid production, also stimulates adrenal androgens. ${ }^{8}$ In a limited number of studies DHEAS in post-surgical patients has been reported and noted significant reductions in their plasma levels..$^{31,32}$ The physiological effects of DHEAS remain poorly understood; however, it is known that normally its blood concentration oscillates in parallel to that of cortisol. ${ }^{8}$ In our study, and in good agreement with the published data for other critically ill patients, ${ }^{9,10}$ early after surgery cortisol and DHEAS levels were dissociated. In fact, it has been suggested that since pregnenolone, derived from cholesterol, is the common precursor to glucocorticoids and adrenal androgens ${ }^{8}$ such a change may represent a shift in pregnenolone metabolism away from androgens and towards increased cortisol production.

However, in a recent study investigating patients with sepsis, measurements of DHEA provided some interesting findings that have questioned the current belief that decreased DHEAS levels occur to enhance adrenal metabolism in favour of cortisol. ${ }^{13}$ These investigators showed that DHEA levels were consistently elevated in septic patients. Our data in patients with major surgery support these findings, since we were able to show a similar dissociation between DHEA (high) and DHEAS (low), suggesting that during biological stress DHEA increases in parallel with cortisol. It has been suggested that such discordance between DHEA (high) and DHEAS (low) may be caused by decreased activity of the SULT2A1 enzyme (that converts DHEA to the biologically active form, DHEAS). ${ }^{33}$

On PoD1, another remarkable dissociation between ACTH (low) and FCI (high) was observed. Such dissociation has been previously described in stressful conditions, such as those caused by surgery ${ }^{3,26,33,34}$ or critical illness. ${ }^{35}$ This phenomenon remains poorly defined and has been attributed to factors which directly stimulate the adrenal or interfere with $\mathrm{CRH}$ and ACTH release. These factors include a variety of cytokines, in particular IL-6, ${ }^{26,36-38}$ or peptides, such as endothelin-1, atrial natriuretic peptide, vasopressin, ${ }^{35}$ 
leptin ${ }^{25}$ and macrophage migration inhibitory factor (MIF). ${ }^{39}$ Further insight into the pathophysiology of dissociated cortisol and ACTH response is offered by the higher response of the adrenals to exogenously administered ACTH after surgery compared to that before surgery. We found that stimulated cortisol on PoD1 was significantly higher compared to that on the day before surgery, suggesting increased adrenal responsiveness. Of note, similar results have been reported by previous investigators on patients undergoing abdominal ${ }^{3}$ or cardiac surgery. ${ }^{40}$ Theoretically, the sharp increase of ACTH on the day of surgery may lead to an increase in adrenal reactivity to ACTH in the immediate postoperative period.

In conclusion, at an early phase following major surgery elevated cortisol is associated with high ACTH. Despite this HPA activation, DHEAS, the major adrenal androgen, is temporarily decreased, whereas DHEA (which represents the biologically active form) is consistently elevated. Free cortisol subsequently remains high while ACTH returns to the pre-operative levels. The enhanced sensitivity of the adrenals to ACTH post-operatively is, at least in part, most likely responsible for maintaining elevated serum cortisol at this stage.

\section{REFERENCES}

1. Desborough JP, 2000, The stress response to trauma and surgery. Br J Anaesth 85: 109-117.

2. Chernow B, Alexander HR, Smallridge RC, et al, 1987 Hormonal responses to graded surgical stress. Arch Intern Med 147: 1273-1278.

3. Naito Y, Fukata J, Tamai S, et al, 1991 Biphasic changes in hypothalamo-pituitary-adrenal function during the early recovery period after major abdominal surgery. $\mathrm{J}$ Clin Endocrinol Metab 73: 111-117.

4. Vogeser M, Felbinger TW, Kilger E, Röll W, Fraunberger P, Jacob K, 1999 Corticosteroid-binding globulin and free cortisol in the early postoperative period after cardiac surgery. Clin Biochem 32: 213-216.

5. Le Roux CW, Chapman GA, Kong WM, Dhillo WS, Jones J, Alaghband-Zadeh J, 2003 Free cortisol index is better than serum total cortisol in determining hypothalamicpituitary-adrenal status in patients undergoing surgery. J Clin Endocrinol Metab 88: 2045-2048.

6. Hamrahian AH, Oseni TS, Arafah BM, 2004 Measurements of serum free cortisol in critically ill patients. $\mathrm{N}$ Engl J Med 350: 1629-1638.

7. Arafah BM, 2006 Hypothalamic pituitary adrenal function during critical illness: limitations of current assessment methods J Clin Endocrinol Metab 91: 3725-3745.

8. Kroboth PD, Salek FS, Pittenger AL, Fabian TJ, Frye RF, 1999 DHEA and DHEA-S: a review. J Clin Pharmacol 39: 327-348.

9. Parker LN, Levin ER, Lifrak ET, 1985 Evidence for adrenocortical adaptation to severe illness. J Clin Endocrinol Metab 60: 947-952.

10. Beishuizen A, Thijs LG, Vermes I, 2002 Decreased levels of dehydroepiandrosterone sulphate in severe critical illness: a sign of exhausted adrenal reserve? Crit Care 6: 434-438.

11. Dhatariya KK, 2003 Is there a role for dehydroepiandrosterone replacement in the intensive care population? Intensive Care Med 29: 1877-1880.

12. Marx C, Petros S, Bornstein SR, et al, 2003 Adrenocortical hormones in survivors and nonsurvivors of severe sepsis: diverse time course of dehydroepiandrosterone, dehydroepiandrosterone-sulfate, and cortisol. Crit Care Med 31: 1382-1388.

13. Arlt W, Hammer F, Sanning P, et al, 2006 Dissociation of serum dehydroepiandrosterone and dehydroepiandrosterone sulfate in septic shock. J Clin Endocrinol Metab 91: 2548-2554.

14. Batrinos ML, Panitsa-Faflia C, Koutsoumanis C, Vourlioti T, Koutsilieris M, 1999 Surgical stress induces a marked and sustained increase of adrenal androgen secretion in postmenopausal women. In Vivo 13: 147-150.

15. Debing E, Peeters E, Duquet W, Poppe K, Velkeniers B, Van den Brande P, 2007 Endogenous sex hormone levels in postmenopausal women undergoing carotid artery endarterectomy. Eur J Endocrinol 156: 687-693.

16. Hassa H, Tanir HM, Ardic N, 2006 Early postoperative changes in testosterone, dehydroepiandrosterone sulfate, and sex hormone-binding globulin after hysterectomy with or without concomitant oophorectomy. Fertil Steril 86: 981-989.

17. Savastano S, Belfiore A, Guida B, et al, 2005 Role of dehydroepiandrosterone sulfate levels on body composition after laparoscopic adjustable gastric banding in pre-menopausal morbidly obese women. J Endocrinol Invest 28: 509-515.

18. Le Roux CW, Sivakumaran S, Alaghband-Zadeh J, Dhillo W, Kong WM, Wheeler MJ, 2002 Free cortisol index as a surrogate marker for serum free cortisol. Ann Clin Biochem 39: 406-408.

19. Dickstein G, Shechner C, Nicholson WE, et al, 1991 Adrenocorticotropin stimulation test: effects of basal cortisol level, time of day, and suggested new sensitive low dose test. J Clin Endocrinol Metab 72: 773-778.

20. Dimopoulou I, Ilias I, Roussou P, et al, 2002 Adrenal function in non-septic long-stay critically ill patients: evaluation with the low-dose ( 1 microg) corticotropin stimulation test. Intensive Care Med 28: 1168-1171.

21. Dimopoulou I, Tsagarakis S, Kouyialis AT, et al, 2004 Hypothalamic-pituitary-adrenal axis dysfunction in critically ill patients with traumatic brain injury: incidence, 
pathophysiology, and relationship to vasopressor dependence and peripheral interleukin-6 levels. Crit Care Med 32: 404-408.

22. Dimopoulou I, Tsagarakis S, Douka E, et al, 2004 The low-dose corticotropin stimulation test in acute traumatic and non-traumatic brain injury: incidence of hypo-responsiveness and relationship to outcome. Intensive Care Med 30: 1216-1219.

23. Oelkers W, 1996 Adrenal insufficiency. N Engl J Med 335: 1206-1212.

24. Borgdorff PJ, Ionescu TI, Houweling PL, Knape JTA 2004 Large-dose intrathecal sufentanil prevents the hormonal stress response during major abdominal surgery: a comparison with intravenous sufentanil in a prospective randomized trial. Anesth Analg 99: 1114-1120.

25. Kain ZN, Zimolo Z, Heninger G, 1999 Leptin and the perioperative neuroendocrinological stress response. J Clin Endocrinol Metab 84: 2438-2442.

26. Cho YM, Kim SY, Cho BY, Lee HK, Yang HK, Lee KU, 2000 Dissociation between plasma adrenocorticotropin and serum cortisol level during the early postoperative period after gastrectomy. Horm Res 53: 246-250.

27. Kato M, Suzuki H, Murakami M, Akama M, Matsukawa S, Hashimoto Y, 1997 Elevated plasma levels of interleukin-6, interleukin-8, and granulocyte colony-stimulating factor during and after major abdominal surgery. Br J Anaesth 9: 293-298.

28. Beishuizen A, Thijs LG, Vermes I, 2001 Patterns of corticosteroid-binding globulin and the free cortisol index during septic shock and multitrauma. Intensive Care Med 27: 1584-1591.

29. Axelrod L, 2003 Perioperative management of patients treated with glucocorticoids. Endocrinol Metab Clin North Am 32: 367-383.

30. Salem M, Tainsh RE, Bromberg J, Loriaux DL, Chernow B, 1994 Perioperative glucocorticoid coverage. A reassessment 42 years after emergence of a problem. Ann
Surg 219: 416-425.

31. Osorio A, Vara-Thorbeck R, Rosell J, Osorio C, Ortega E, Ruiz-Requena E, 2002 Dehydroepiandrosterone sulfate and growth axis hormones in patients after surgery. World J Surg 26: 1079-1082.

32. Stahl F, Schnorr D, Pilz C, Dörner G, 1992 Dehydroepiandrosterone (DHEA) levels in patients with prostatic cancer, heart diseases and under surgery stress. Exp Clin Endocrinol 99: 68-70.

33. Kim MS, Shigenaga J, Moser A, Grunfeld C, Feingold KR, 2004 Suppression of DHEA sulfotransferase (Sult2A1) during the acute-phase response. Am J Physiol Endocrinol Metab 287: E731-E738.

34. Roth-Isigkeit AK, Schmucker P, 1997 Postoperative dissociation of blood levels of cortisol and adrenocorticotropin after coronary artery bypass grafting surgery. Steroids 62: 695-699.

35. Vermes I, Beishuizen A, Hampsink RM, Haanen C, 1995 Dissociation of plasma adrenocorticotropin and cortisol levels in critically ill patients: possible role of endothelin and atrial natriuretic hormone. J Clin Endocrinol Metab 80: $1238-1242$.

36. Turnbull AV, Rivier CL, 1999 Regulation of the hypothalamic-pituitary-adrenal axis by cytokines: actions and mechanisms of action. Physiol Rev 79: 1-71.

37. Tsagarakis S, Kontogeorgos G, Kovacs K, 1998 The role of cytokines in the normal and neoplastic pituitary. Crit Rev Oncol Hematol 28: 73-90.

38. Bornstein SR, Rutkowski H, Vrezas I, 2004 Cytokines and steroidogenesis. Mol Cell Endocrinol 215: 135-141.

39. Beishuizen A, Thijs LG, Haanen C, Vermes I, 2001 Macrophage migration inhibitory factor and hypothalamopituitary-adrenal function during critical illness. J Clin Endocrinol Metab 86: 2811-2816.

40. Widmer IE, Puder JJ, König C, et al, 2005 Cortisol response in relation to the severity of stress and illness. J Clin Endocrinol Metab 90: 4579-4586. 